\title{
Liquid Chromatography Column Theory and Technology
}

\section{Lane C. Sander}

National Institute of Standards and Technology, Gaithersburg, MD 20899, USA

lane.sander@nist.gov

Video DOI: http://doi.org/10.18434/T4DK5T

Key words: selectivity; stationary phase; modified surfaces; reversed-phase; normal-phase; core-shell columns.

Accepted: March 6, 2017

Published: March 8, 2017

https://doi.org/10.6028/jres.122.017

\section{Summary}

Column selection is probably the single most important aspect of method development that will directly affect measurement accuracy and precision of liquid chromatographic (LC) measurements. An understanding of physical and chemical properties of LC columns will greatly assist the development and optimization of robust analytical methods. This presentation will describe aspects of the preparation, characterization, and use of different types of liquid chromatographic columns, to facilitate informed selection of columns for the development of new methods. Topics include: substrates, synthesis of bonded stationary phases, spectroscopic approaches to characterize covalently modified surfaces, chromatographic retention mechanisms for normal-phase and reversed-phase separations, and properties of the most common types of commercially available columns, including newly developed core-shell technologies. ${ }^{1}$

\footnotetext{
${ }^{1}$ Contribution of the National Institute of Standards and Technology. Not subject to copyright. Certain commercial equipment, instruments, or materials are identified to specify adequately the experimental procedure. Such identification does not imply recommendation or endorsement by the National Institute of Standards and Technology, nor does it imply that the materials or equipment identified are the best available for the purpose.
} 